\title{
Glymour and Quine on Theoretical Equivalence*
}

\author{
Thomas William Barrett ${ }^{\dagger}$ \\ Hans Halvorson ${ }^{\ddagger}$
}

\begin{abstract}
Glymour (1970, 1977, 1980) and Quine (1975) propose two different formal criteria for theoretical equivalence. In this paper we examine the relationships between these criteria.
\end{abstract}

\section{Introduction}

Philosophers of science have long been concerned with the conditions under which theories might be considered equivalent. ${ }^{1}$ One way that this issue has been approached is by proposing different formal criteria for theoretical equivalence. In this paper we will discuss two such criteria. The first was proposed by Glymour $(1970,1977,1980)$ and the second was proposed by Quine (1975).

We begin by showing that Quine's criterion is unsatisfactory. It considers some theories to be equivalent that one has good reason to consider inequivalent. But Quine's criterion can be amended in such a way that it no longer makes these undesirable verdicts. Indeed, we will isolate a precise sense in which Glymour's criterion is such an amendment.

\section{Preliminaries}

The criteria for theoretical equivalence that we will discuss are most naturally understood in the framework of first-order logic. We therefore begin by presenting some preliminaries about this framework. ${ }^{2}$

\footnotetext{
${ }^{*}$ Thanks to JB Manchak, Jim Weatherall, Jeff Barrett, and Albert Visser for comments and discussion.

†thomaswb@princeton.edu

‡hhalvors@princeton.edu

${ }^{1}$ For example, North (2009), Halvorson (2011), Swanson and Halvorson (2012), Curiel (2014), and Barrett (2014) discuss whether Hamiltonian and Lagrangian mechanics are theoretically equivalent. Glymour (1977), Weatherall (2014), and Knox (2013) discuss standard Newtonian gravitation and geometrized Newtonian gravitation. And Sklar (1982), Halvorson (2012, 2013), Glymour (2013), van Fraassen (2014), and Coffey (2014) discuss more general issues about theoretical equivalence.

${ }^{2}$ The reader is encouraged to consult Hodges (2008) for details and notation.
} 
A signature $\Sigma$ is a set of predicate symbols, function symbols, and constant symbols. The $\Sigma$-terms, $\Sigma$-formulas, and $\Sigma$-sentences are recursively defined in the standard way. A $\Sigma$-structure $A$ is a nonempty set in which the symbols of $\Sigma$ have been interpreted. One recursively defines when a sequence of elements $a_{1}, \ldots, a_{n} \in A$ satisfy a $\Sigma$-formula $\phi\left(x_{1}, \ldots, x_{n}\right)$ in a $\Sigma$-structure $A$, written $A \vDash \phi\left[a_{1}, \ldots, a_{n}\right]$. If $\phi$ is a $\Sigma$-sentence, then $A \vDash \phi$ just in case the empty sequence satisfies $\phi$ in $A$.

A $\boldsymbol{\Sigma}$-theory $T$ is a set of $\Sigma$-sentences. The sentences $\phi \in T$ are called the axioms of $T$. A $\Sigma$-structure $M$ is a model of a $\Sigma$-theory $T$ if $M \vDash \phi$ for all $\phi \in T$. We will use the notation $\operatorname{Mod}(T)$ to denote the class of models of a theory $T$. A theory $T$ entails a sentence $\phi$, written $T \vDash \phi$, if $M \vDash \phi$ for every model $M$ of $T$.

We begin with the following preliminary criterion for theoretical equivalence.

Definition. Theories $T_{1}$ and $T_{2}$ are logically equivalent if they have the same class of models, i.e. if $\operatorname{Mod}\left(T_{1}\right)=\operatorname{Mod}\left(T_{2}\right)$.

One can easily verify that $T_{1}$ and $T_{2}$ are logically equivalent if and only if $\left\{\phi: T_{1} \vDash \phi\right\}=\left\{\psi: T_{2} \vDash \psi\right\}$. Note that logical equivalence can only apply to theories $T_{1}$ and $T_{2}$ that are formulated in the same signature.

\section{Glymour and Quine}

Although we have the notion of logical equivalence, one might want other criteria for theoretical equivalence. Logical equivalence is too strict to capture the sense in which some theories are equivalent. Indeed, as remarked above, theories can be logically equivalent only if they are formulated in the same signature. And there are many theories in different signatures that are nonetheless equivalent in some sense. For example, group theory can be formulated in different signatures.

Example 1. Let $\Sigma_{1}=\{\cdot, e\}$ be a signature where $\cdot$ is a binary function symbol and $e$ is a constant symbol. The theory of groups ${ }_{1}$ is the following $\Sigma_{1}$-theory:

$$
\begin{gathered}
\{\forall x \forall y \forall z((x \cdot y) \cdot z=x \cdot(y \cdot z)), \forall x(x \cdot e=x \wedge e \cdot x=x), \\
\forall x \exists y(x \cdot y=e \wedge y \cdot x=e)\}
\end{gathered}
$$

Group theory can also be formulated in the signature $\Sigma_{2}=\{\cdot,-1\}$, where $\cdot$ is again a binary function symbol and -1 is a unary function symbol. The theory of groups ${ }_{2}$ is the following $\Sigma_{2}$-theory:

$$
\begin{gathered}
\{\forall x \forall y \forall z((x \cdot y) \cdot z=x \cdot(y \cdot z)), \\
\left.\exists x \forall y\left(y \cdot x=y \wedge x \cdot y=y \wedge y \cdot y^{-1}=x \wedge y^{-1} \cdot y=x\right)\right\}
\end{gathered}
$$

One can easily see that these two theories are not logically equivalent. A model $M$ of the theory of groups 1 is a set with a binary function ${ }^{M}$ and a distinguished element $e^{M}$. A model $N$ of the theory of groups 2 is a set with a binary function .$^{N}$ and a unary function $-1^{N}$. These are not the same, so the theories do not have the same class of models. 
If one wants to capture the sense in which these two formulations of group theory are equivalent, then one needs a more general criterion for theoretical equivalence than logical equivalence. Glymour and Quine's proposals are two candidates for such a criterion.

\subsection{Glymour's criterion}

Glymour $(1970,1977,1980)$ proposed definitional equivalence as a criterion for theoretical equivalence. ${ }^{3}$ The basic idea behind definitional equivalence is simple. Theories $T_{1}$ and $T_{2}$ are definitionally equivalent if $T_{1}$ can define all of the vocabulary that $T_{2}$ uses, and in a compatible way, $T_{2}$ can define all of the vocabulary that $T_{1}$ uses. In order to state this criterion carefully we need to do some work.

We first need to formalize the notion of a definition. Let $\Sigma \subset \Sigma^{+}$be signatures and let $p \in \Sigma^{+}-\Sigma$ be an $n$-ary predicate symbol. An explicit definition of $p$ in terms of $\Sigma$ is a $\Sigma^{+}$-sentence of the form

$$
\forall x_{1} \ldots \forall x_{n}\left(p\left(x_{1}, \ldots, x_{n}\right) \leftrightarrow \phi\left(x_{1}, \ldots, x_{n}\right)\right)
$$

where $\phi\left(x_{1}, \ldots, x_{n}\right)$ is a $\Sigma$-formula. Similarly, an explicit definition of an $n$-ary function symbol $f \in \Sigma^{+}-\Sigma$ is a $\Sigma^{+}$-sentence of the form

$$
\forall x_{1} \ldots \forall x_{n} \forall y\left(f\left(x_{1}, \ldots, x_{n}\right)=y \leftrightarrow \phi\left(x_{1}, \ldots, x_{n}, y\right)\right)
$$

and an explicit definition of a constant symbol $c \in \Sigma^{+}-\Sigma$ is a $\Sigma^{+}$-sentence of the form

$$
\forall x(x=c \leftrightarrow \psi(x))
$$

where $\phi\left(x_{1}, \ldots, x_{n}, y\right)$ and $\psi(x)$ are both $\Sigma$-formulas.

Although they are $\Sigma^{+}$-sentences, (1) and (2) have consequences in the signature $\Sigma$. In particular, (1) and (2) imply the following sentences, respectively:

$$
\begin{gathered}
\forall x_{1} \ldots \forall x_{n} \exists_{=1} y \phi\left(x_{1}, \ldots, x_{n}, y\right) \\
\exists_{=1} x \psi(x)
\end{gathered}
$$

These two sentences are called the admissibility conditions for the explicit definitions (1) and (2).

A definitional extension of a $\Sigma$-theory $T$ to the signature $\Sigma^{+}$is a $\Sigma^{+}$theory

$$
T^{+}=T \cup\left\{\delta_{s}: s \in \Sigma^{+}-\Sigma\right\},
$$

that satisfies the following two conditions. First, for each symbol $s \in \Sigma^{+}-\Sigma$ the sentence $\delta_{s}$ is an explicit definition of $s$ in terms of $\Sigma$, and second, if $s$ is a constant symbol or a function symbol and $\alpha_{s}$ is the admissibility condition for $\delta_{s}$ then $T \vDash \alpha_{s}$.

We now have the machinery necessary to state Glymour's criterion.

\footnotetext{
${ }^{3}$ Logicians were familiar with definitional equivalence before the 1970s, but Glymour was the first to introduce the notion into philosophy of science.
} 
Definition. Let $T_{1}$ be a $\Sigma_{1}$-theory and $T_{2}$ be a $\Sigma_{2}$-theory. $T_{1}$ and $T_{2}$ are definitionally equivalent if there is a definitional extension $T_{1}^{+}$of $T_{1}$ to the signature $\Sigma_{1} \cup \Sigma_{2}$ and a definitional extension $T_{2}^{+}$of $T_{2}$ to the signature $\Sigma_{1} \cup \Sigma_{2}$ such that $T_{1}^{+}$and $T_{2}^{+}$are logically equivalent.

One often says that $T_{1}$ and $T_{2}$ are definitionally equivalent if they have a "common definitional extension."

Definitional equivalence captures a sense in which theories formulated in different signatures might nonetheless be theoretically equivalent. For example, although they are not logically equivalent, the theory of groups ${ }_{1}$ and the theory of groups 2 are definitionally equivalent.

Example 2. Recall the two formulations of group theory from Example 1 and consider the following two $\Sigma_{1} \cup \Sigma_{2}$-sentences.

$$
\begin{aligned}
\delta_{-1} & :=\forall x \forall y\left(x^{-1}=y \leftrightarrow(x \cdot y=e \wedge y \cdot x=e)\right) \\
\delta_{e} & :=\forall x(x=e \leftrightarrow \forall z(z \cdot x=z \wedge x \cdot z=z))
\end{aligned}
$$

The theory of groups 1 defines the unary function symbol -1 with the sentence $\delta_{-1}$ and the theory of groups 2 defines the constant symbol $e$ with the sentence $\delta_{e}$. One can verify that the theory of groups ${ }_{1}$ satisfies the admissibility condition for $\delta_{-1}$ and that the theory of groups 2 satisfies the admissibility condition for $\delta_{e}$. The theory of groups $\cup\left\{\delta_{-1}\right\}$ and the theory of groups ${ }_{2} \cup\left\{\delta_{e}\right\}$ are logically equivalent. This implies that these two formulations of group theory are definitionally equivalent.

Definitional equivalence is well-known among logicians, and many results about it have been proven. ${ }^{4}$ Here we state one particular fact that will be useful in what follows. Let $\Sigma \subset \Sigma^{+}$be signatures. A $\Sigma^{+}$-theory $T^{+}$is a conservative extension of a $\Sigma$-theory $T$ if for every $\Sigma$-sentence $\phi, T^{+} \vDash \phi$ if and only if $T \vDash \phi$.

Proposition 1. If $T^{+}$is a definitional extension of $T$, then $T^{+}$is a conservative extension of $T$ (Hodges, 2008, 66).

\subsection{Quine's criterion}

The criterion that Quine (1975) proposes is of a different flavor than Glymour's criterion. Quine suggests that two theories should be considered theoretically equivalent if there is a "suitable translation" between the theories. In order to state Quine's criterion carefully we again need to do some work.

We begin by introducing the idea of a reconstrual between signatures $\Sigma_{1}$ and $\Sigma_{2}$. A reconstrual $F$ of $\Sigma_{1}$ into $\Sigma_{2}$ is a map from elements of the signature $\Sigma_{1}$ to $\Sigma_{2}$-formulas that satisfies the following three conditions.

\footnotetext{
${ }^{4}$ For example, see (Hodges, 2008, 58-62), de Bouvére (1965), Kanger (1968), Pelletier and Urquhart (2003), Andréka et al. (2005), Friedman and Visser (2014) for some results.
} 
- For every $n$-ary predicate symbol $p \in \Sigma_{1}, F p\left(x_{1}, \ldots, x_{n}\right)$ is a $\Sigma_{2}$-formula with $n$ free variables.

- For every $n$-ary function symbol $f \in \Sigma_{1}, F f\left(x_{1}, \ldots, x_{n}, y\right)$ is a $\Sigma_{2}$-formula with $n+1$ free variables.

- For every constant symbol $c \in \Sigma_{1}, F c(y)$ is a $\Sigma_{2}$-formula with one free variable.

One can think of the $\Sigma_{2}$-formula $F p\left(x_{1}, \ldots, x_{n}\right)$ as a "translation" of the $\Sigma_{1^{-}}$ formula $p\left(x_{1}, \ldots, x_{n}\right)$ into the signature $\Sigma_{2}$. Similarly, $F f\left(x_{1}, \ldots, x_{n}, y\right)$ and $F c(y)$ can be thought of as "translations" of the $\Sigma_{1}$-formulas $f\left(x_{1}, \ldots, x_{n}\right)=y$ and $c=y$, respectively. We will use the notation $F: \Sigma_{1} \rightarrow \Sigma_{2}$ to denote a reconstrual $F$ of $\Sigma_{1}$ into $\Sigma_{2}$.

Before stating Quine's criterion we need to take a moment to discuss reconstruals. The important fact about a reconstrual $F: \Sigma_{1} \rightarrow \Sigma_{2}$ is that it naturally induces a map from $\Sigma_{1}$-formulas to $\Sigma_{2}$ formulas.

In order to describe this map we first need to describe what $F$ does to $\Sigma_{1}$ terms. $F$ extends to a map from $\Sigma_{1}$-terms to $\Sigma_{2}$-formulas. Let $t\left(x_{1}, \ldots, x_{n}\right)$ be a $\Sigma_{1}$-term. We define the $\Sigma_{2}$-formula $F t\left(x_{1}, \ldots, x_{n}, y\right)$ recursively as follows.

- If $t$ is the variable $x_{i}$ then $F t\left(x_{i}, y\right)$ is the $\Sigma_{2}$-formula $x_{i}=y$.

- If $t$ is the constant symbol $c \in \Sigma_{1}$ then $F t(y)$ is the $\Sigma_{2}$-formula $F c(y)$.

- Suppose that $t$ is the term $f\left(t_{1}\left(x_{1}, \ldots, x_{n}\right), \ldots, t_{k}\left(x_{1}, \ldots, x_{n}\right)\right)$ and that the $\Sigma_{2}$-formula $F t_{i}\left(x_{1}, \ldots, x_{n}, y\right)$ has been defined for each $i=1, \ldots, k$. Then $F t\left(x_{1}, \ldots x_{n}, y\right)$ is the $\Sigma_{2}$-formula

$$
\exists z_{1} \ldots \exists z_{k}\left(F t_{1}\left(x_{1}, \ldots, x_{n}, z_{1}\right) \wedge \ldots \wedge F t_{k}\left(x_{1}, \ldots, x_{n}, z_{k}\right) \wedge F f\left(z_{1}, \ldots, z_{k}, y\right)\right)
$$

Using this induced map from $\Sigma_{1}$-terms to $\Sigma_{2}$-formulas, we can describe how $F$ maps $\Sigma_{1}$-formulas to $\Sigma_{2}$-formulas. ${ }^{5}$ Let $\phi\left(x_{1}, \ldots, x_{n}\right)$ be a $\Sigma_{1}$-formula. We define the $\Sigma_{2}$-formula $F \phi\left(x_{1}, \ldots, x_{n}\right)$ recursively as follows.

- If $\phi\left(x_{1}, \ldots, x_{n}\right)$ is the $\Sigma_{1}$-atom $s\left(x_{1}, \ldots, x_{n}\right)=t\left(x_{1}, \ldots, x_{n}\right)$, with $s$ and $t \Sigma_{1}$ terms, then $F \phi\left(x_{1}, \ldots, x_{n}\right)$ is the $\Sigma_{2}$-formula

$$
\exists z\left(F t\left(x_{1}, \ldots, x_{n}, z\right) \wedge F s\left(x_{1}, \ldots, x_{n}, z\right)\right)
$$

- If $\phi\left(x_{1}, \ldots, x_{n}\right)$ is the $\Sigma_{1}$-atom $p\left(t_{1}\left(x_{1}, \ldots, x_{n}\right), \ldots, t_{k}\left(x_{1}, \ldots, x_{n}\right)\right)$, with $p \in \Sigma_{1}$ a $k$-ary predicate symbol, then $F \phi\left(x_{1}, \ldots, x_{n}\right)$ is the $\Sigma_{2}$-formula

$$
\exists z_{1} \ldots \exists z_{k}\left(F t_{1}\left(x_{1}, \ldots, x_{n}, z_{1}\right) \wedge \ldots \wedge F t_{k}\left(x_{1}, \ldots, x_{n}, z_{k}\right) \wedge F p\left(z_{1}, \ldots, z_{k}\right)\right)
$$

\footnotetext{
${ }^{5}$ We are abusing notation by calling all of these maps $F$. In what follows, the important map is the map $F$ from $\Sigma_{1}$-formulas to $\Sigma_{2}$-formulas. Whenever we refer to a reconstrual $F: \Sigma_{1} \rightarrow \Sigma_{2}$ from now on, we will be referring to this induced map from $\Sigma_{1}$-formulas to $\Sigma_{2}$-formulas.
} 
- Lastly, suppose that $F \phi\left(x_{1}, \ldots, x_{n}\right)$ and $F \psi\left(x_{1}, \ldots, x_{n}\right)$ have already been defined for $\Sigma_{1}$-formulas $\phi\left(x_{1}, \ldots, x_{n}\right)$ and $\psi\left(x_{1}, \ldots, x_{n}\right)$. Then we define $F \neg \phi$ to be $\neg F \phi, F(\phi \wedge \psi)$ to be $F(\phi) \wedge F(\psi), F(\phi \rightarrow \psi)$ to be $F(\phi) \rightarrow$ $F(\psi), F(\phi \vee \psi)$ to be $F(\phi) \vee F(\psi), F(\phi \leftrightarrow \psi)$ to be $F(\phi) \leftrightarrow F(\psi), F(\forall x \phi)$ to be $\forall x F(\phi)$, and $F(\exists x \phi)$ to be $\exists x F(\phi)$.

In this way a reconstrual $F: \Sigma_{1} \rightarrow \Sigma_{2}$ gives rise to a map between $\Sigma_{1}$-formulas and $\Sigma_{2}$-formulas. This map allows one to "translate" $\Sigma_{1}$-theories into $\Sigma_{2^{-}}$ theories. If $T_{1}$ is a $\Sigma_{1}$ theory and $F: \Sigma_{1} \rightarrow \Sigma_{2}$ a reconstrual, then the $\Sigma_{2}$-theory $F\left(T_{1}\right)$ is defined by

$$
F\left(T_{1}\right)=\left\{F(\phi): \phi \in T_{1}\right\}
$$

We now have the machinery necessary to state Quine's criterion for theoretical equivalence. ${ }^{6}$

Definition. Let $T_{1}$ be a $\Sigma_{1}$-theory and $T_{2}$ a $\Sigma_{2}$-theory. $T_{1}$ is Quine equivalent to $T_{2}$ if there is a reconstrual $F: \Sigma_{1} \rightarrow \Sigma_{2}$ such that the theories $F\left(T_{1}\right)$ and $T_{2}$ are logically equivalent.

At first glance, Quine equivalence seems to be a promising way to understand theoretical equivalence. And indeed, one can use it to capture a sense in which the theory of groups 1 is equivalent to the theory of groups 2 .

Example 3. Recall again the theory of groups $s_{1}$ and the theory of groups $s_{2}$. We define a reconstrual $F: \Sigma_{1} \rightarrow \Sigma_{2}$ by letting $F \cdot\left(x_{1}, x_{2}, y\right)$ be the $\Sigma_{2}$-formula $x_{1} \cdot x_{2}=y$ and $F e(y)$ be the $\Sigma_{2}$-formula $\forall z\left(y \cdot z=z \wedge z \cdot y=z \wedge z \cdot z^{-1}=\right.$ $y \wedge z^{-1} \cdot z=y$ ). One can then verify that the $\Sigma_{2}$-theory $F$ (theory of groups ${ }_{1}$ ) is logically equivalent to the theory of groups 2 . The theory of groups ${ }_{1}$ and the theory of groups 2 are therefore Quine equivalent.

\subsection{Problems with Quine equivalence}

Unlike definitional equivalence, Quine equivalence has not yet been investigated by logicians. ${ }^{7}$ And upon investigation one finds that Quine equivalence is unsatisfactory. The following two examples illustrate some of its shortcomings.

These examples show that Quine equivalence is too liberal a criterion for theoretical equivalence. It considers some theories to be equivalent that one has good reason to consider inequivalent.

\footnotetext{
${ }^{6}$ Quine explains his proposal as follows: "By a reconstrual of the predicates of our language, accordingly, let me mean any mapping of our lexicon of predicates into our open sentences ( $n$-place predicates to $n$-variable sentences). [...] I propose to individuate theories thus: two formulations express the same theory if they are empirically equivalent and there is a reconstrual of predicates that transforms the one theory into a logical equivalent of the other" (Quine, 1975, 320).

${ }^{7}$ As of February 24, 2015, according to scholar.google.com, there have been no technical investigations of Quine's proposal.
} 
Example 4. Let $\Sigma=\{p, q\}$ be a signature with $p$ and $q$ both unary predicate symbols. Consider the following two $\Sigma$-theories.

$$
\begin{aligned}
& T_{1}=\left\{\exists_{=1} x(x=x), \forall x(p(x) \wedge q(x))\right\} \\
& T_{2}=\left\{\exists_{=1} x(x=x), \forall x p(x)\right\}
\end{aligned}
$$

The theory $T_{2}$ is Quine equivalent to the theory $T_{1}$. We define the reconstrual $F: \Sigma \rightarrow \Sigma$ by letting $F p(x)$ be $p(x) \wedge q(x)$ and letting $F q(x)$ be $q(x)$. One can then easily verify that the following hold.

$$
\begin{gathered}
F\left(\exists_{=1} x(x=x)\right) \text { is equivalent to } \exists_{=1} x(x=x) \\
F(\forall x(p(x)) \text { is equivalent to } \forall x(p(x) \wedge q(x))
\end{gathered}
$$

This implies that $F\left(T_{2}\right)$ is logically equivalent to $T_{1}$.

$T_{2}$ is Quine equivalent to $T_{1}$, and that is a strange verdict. In fact, one can make precise a sense in which $T_{1}$ and $T_{2}$ are inequivalent theories. We call a $\Sigma$-theory $T$ complete if either $T \vDash \phi$ or $T \vDash \neg \phi$ for every $\Sigma$-sentence $\phi$. Consider the theory $T_{1}$ from the above example. Every model $M$ of $T_{1}$ has a domain with one element, and this one element is in both the sets $p^{M}$ and $q^{M}$. So up to isomorphism, $T_{1}$ has a unique model. Since this model $M$ satisfies either $M \vDash \phi$ or $M \vDash \neg \phi$ for every $\Sigma$-sentence $\phi$, it must also be that $T \vDash \phi$ or $T \vDash \neg \phi$ for every $\Sigma$-sentence $\phi$. The theory $T_{1}$ is therefore complete. But the theory $T_{2}$ is not complete. Consider the $\Sigma$-sentence $\forall x q(x)$. One can easily see that $T_{2} \not \forall \forall x q(x)$ and $T_{2} \not \forall \neg \forall x q(x)$. $T_{1}$ is Quine equivalent to $T_{2}$, but these two theories are inequivalent in a precise sense: $T_{1}$ is complete and $T_{2}$ is not.

Example 4 shows that completeness is not an "invariant" under Quine equivalence, and this might strike one as a shortcoming Quine equivalence. The following example provides another case where Quine equivalence makes a puzzling verdict.

Example 5. Let $\Sigma_{1}=\emptyset$ and $\Sigma_{2}=\{c, d\}$ be signatures with $c$ and $d$ constant symbols. Consider the $\Sigma_{1}$-theory $T_{1}=\emptyset$ and the $\Sigma_{2}$-theory $T_{2}=\{c=d\}$. The theory $T_{2}$ is Quine equivalent to the theory $T_{1}$. We define the reconstrual $G: \Sigma_{2} \rightarrow \Sigma_{1}$ by letting both $G c(y)$ and $G d(y)$ be the $\Sigma_{1}$-formula $y=y$. One can then easily see that

$$
G(c=d) \text { is equivalent to } \exists z(z=z)
$$

It follows that $G\left(T_{2}\right)$ is logically equivalent to $T_{1}$, so $T_{2}$ is Quine equivalent to $T_{1}$.

There are two things to notice about Example 5. First, note that Quine equivalence is again making a strange verdict. There is a sense in which $T_{1}$ and $T_{2}$ are inequivalent theories. The theory $T_{2}$ uses the constant symbols $c$ and $d$ to single out a preferred point in every model. The theory $T_{1}$ does not single out a preferred point in any model. For this reason one might not want to consider these two theories equivalent. 
Second, note that although $T_{2}$ is Quine equivalent to $T_{1}, T_{1}$ is not Quine equivalent to $T_{2}$. There is a unique reconstrual $F: \Sigma_{1} \rightarrow \Sigma_{2}$, the "empty reconstrual." The theory $F\left(T_{1}\right)$ is the empty theory in the signature $\Sigma_{2}$. And so $F\left(T_{1}\right)$ is not logically equivalent to $T_{2}$. This implies that Quine equivalence is not a symmetric relation on theories. ${ }^{8}$

Neither of these examples pose a problem for definitional equivalence. The two theories from Example 4 are not definitionally equivalent, and neither are the two theories from Example 5. Quine equivalence, however, makes puzzling verdicts in both of these cases.

\section{Intertranslatability}

One might wonder whether there is a way to amend Quine's original proposal so that it no longer makes these undesirable verdicts. The basic idea behind Quine equivalence is that two theories should count as theoretically equivalent if there exists a "suitable translation" between them. Definitional equivalence is often thought of as imposing a similar requirement. For example, Glymour (1970) remarks that definitional equivalence captures the idea that two theories are "intertranslatable." ${ }^{9}$ In this final section we make this remark precise, and in doing so, we show that definitional equivalence can itself be understood as an amendment to Quine's original proposal.

The way that Quine explicates the notion of a "suitable translation" between theories makes Quine equivalence too liberal a criterion for theoretical equivalence. But one can be more restrictive about what counts as a "suitable translation" than Quine is. We call a reconstrual $F: \Sigma_{1} \rightarrow \Sigma_{2}$ a translation of a $\Sigma_{1}$-theory $T_{1}$ into a $\Sigma_{2}$-theory $T_{2}$ if $T_{1} \vDash \phi$ implies that $T_{2} \vDash F \phi$ for all $\Sigma_{1}$-sentences $\phi$. We will use the notation $F: T_{1} \rightarrow T_{2}$ to denote a translation of $T_{1}$ into $T_{2}$.

We can then consider an amendment to Quine's criterion, which we call "intertranslatability."

Definition. Let $T_{1}$ be a $\Sigma_{1}$-theory and $T_{2}$ a $\Sigma_{2}$-theory. $T_{1}$ and $T_{2}$ are intertranslatable if there are translations $F: T_{1} \rightarrow T_{2}$ and $G: T_{2} \rightarrow T_{1}$ such that

$$
\begin{aligned}
T_{1} \vDash \forall x_{1} \ldots \forall x_{n}\left(\phi\left(x_{1}, \ldots, x_{n}\right) \leftrightarrow G F \phi\left(x_{1}, \ldots, x_{n}\right)\right) \\
T_{2} \vDash \forall x_{1} \ldots \forall x_{n}\left(\psi\left(x_{1}, \ldots, x_{n}\right) \leftrightarrow F G \psi\left(x_{1}, \ldots, x_{n}\right)\right)
\end{aligned}
$$

for every $\Sigma_{1}$-formula $\phi\left(x_{1}, \ldots, x_{n}\right)$ and every $\Sigma_{2}$-formula $\psi\left(x_{1}, \ldots, x_{n}\right)$.

The conditions (3) and (4) can be thought of as requiring the translations $F$ : $T_{1} \rightarrow T_{2}$ and $G: T_{2} \rightarrow T_{1}$ to be "almost inverse" to one another. Note, however,

\footnotetext{
${ }^{8}$ Coffey (2014) argues that symmetry is not a good feature for a proposed criterion for theoretical equivalence to have. But both Coffey and Quine suggest that Quine equivalence is an equivalence relation. We have shown here that this is not the case.

${ }^{9}$ Knox (2013) and Coffey (2014) make this same remark.
} 
that $F$ and $G$ need not be literal inverses. The $\Sigma_{1}$-formula $G F \phi\left(x_{1}, \ldots, x_{n}\right)$ is not required to be equal to the $\Sigma_{1}$-formula $\phi\left(x_{1}, \ldots, x_{n}\right)$. Rather, these two formulas are merely required to be equivalent according to the theory $T_{1}$.

Like Quine's original proposal, intertranslatability judges theories to be equivalent when "suitable translations" exist between them. It is just more restrictive about what should count as a "suitable translation." Our goal in the remainder of this section is to show how intertranslatability relates to definitional equivalence. The following theorem provides a partial answer to this question. ${ }^{10}$

Theorem 1. If $T_{1}$ and $T_{2}$ are definitionally equivalent, then they are intertranslatable.

Proof. Suppose that $T$ is a common definitional extension of a $\Sigma_{1}$-theory $T_{1}$ and a $\Sigma_{2}$-theory $T_{2}$. We define a reconstrual $F: \Sigma_{1} \rightarrow \Sigma_{2}$ as follows. Let $p \in \Sigma_{1}$ be an $n$-ary predicate symbol. Then since $T$ is a definitional extension of $T_{2}$,

$$
T \vDash \forall x_{1} \ldots \forall x_{n}\left(p\left(x_{1}, \ldots, x_{n}\right) \leftrightarrow \delta_{p}\left(x_{1}, \ldots, x_{n}\right)\right)
$$

for some $\Sigma_{2}$-formula $\delta_{p}\left(x_{1}, \ldots, x_{n}\right)$. We define $F p\left(x_{1}, \ldots, x_{n}\right)$ to be the $\Sigma_{2^{-}}$ formula $\delta_{p}\left(x_{1}, \ldots, x_{n}\right)$. Let $f \in \Sigma_{1}$ be an $n$-ary function symbol. We define $F f\left(x_{1}, \ldots, x_{n}, y\right)$ to be $\delta_{f}\left(x_{1}, \ldots, x_{n}, y\right)$, where $\delta_{f}$ is the formula that $T$ uses to define the function symbol $f$. Lastly, let $c \in \Sigma_{1}$ be a constant symbol. We again define $F c(y)$ to be $\delta_{c}(y)$. A reconstrual $G: \Sigma_{2} \rightarrow \Sigma_{1}$ is defined in the analogous way. One can then verify by induction on complexity that

$$
\begin{aligned}
T \vDash \forall x_{1} \ldots \forall x_{n}\left(\phi\left(x_{1}, \ldots, x_{n}\right) \leftrightarrow F \phi\left(x_{1}, \ldots, x_{n}\right)\right) \\
T \vDash \forall x_{1} \ldots \forall x_{n}\left(\psi\left(x_{1}, \ldots, x_{n}\right) \leftrightarrow G \psi\left(x_{1}, \ldots, x_{n}\right)\right)
\end{aligned}
$$

for every $\Sigma_{1}$-formula $\phi\left(x_{1}, \ldots, x_{n}\right)$ and every $\Sigma_{2}$-formula $\psi\left(x_{1}, \ldots, x_{n}\right)$.

We need to show that $F: \Sigma_{1} \rightarrow \Sigma_{2}$ and $G: \Sigma_{2} \rightarrow \Sigma_{1}$ are translations. Without loss of generality we show that $F$ is. Suppose that $T_{1} \vDash \phi$ for some $\Sigma_{1}$ sentence $\phi$. Then by equation (5) above, $T \vDash \phi \leftrightarrow F \phi$. Proposition 1 guarantees that $T \vDash \phi$, so it must be that $T \vDash F \phi$. Since $F \phi$ is a $\Sigma_{2}$-sentence, Proposition 1 then implies that $T_{2} \vDash F \phi$. So $F: T_{1} \rightarrow T_{2}$ is a translation.

Now let $\phi\left(x_{1}, \ldots, x_{n}\right)$ be any $\Sigma_{1}$-formula. Equations (5) and (6) together imply that $T \vDash \forall x_{1} \ldots \forall x_{n}\left(\phi\left(x_{1}, \ldots, x_{n}\right) \leftrightarrow G F \phi\left(x_{1}, \ldots, x_{n}\right)\right)$. Since $T$ is a conservative extension of $T_{1}$ by Proposition 1 , it must be that

$$
T_{1} \vDash \forall x_{1} \ldots \forall x_{n}\left(\phi\left(x_{1}, \ldots, x_{n}\right) \leftrightarrow G F \phi\left(x_{1}, \ldots, x_{n}\right)\right)
$$

\footnotetext{
${ }^{10}$ Glymour (1970) remarks that definitional equivalence "guarantees that all and only theorems of $\left[T_{1}\right]$ are translated as theorems of $\left[T_{2}\right]$, and conversely" (Glymour, 1970, 279). But he also claims that requiring the conditions (3) and (4) above to hold is "stronger" than definitional equivalence. Theorems 1 and 2 make precise a sense in which this requirement is no stronger and no weaker than definitional equivalence. Friedman and Visser (2014) state these two results, but do not provide proofs. Ingredients for proofs using tools of category theory are contained in Visser (2006). Pelletier and Urquhart (2003) provide proofs for the special case of propositional logic. Here we extend the results to full first-order logic using only elementary methods.
} 
A similar argument yields $T_{2} \vDash \forall x_{1} \ldots \forall x_{n}\left(\psi\left(x_{1}, \ldots, x_{n}\right) \leftrightarrow F G \psi\left(x_{1}, \ldots, x_{n}\right)\right)$ for every $\Sigma_{2}$-formula $\psi\left(x_{1}, \ldots, x_{n}\right)$.

This result immediately provides us with many examples of theories that are intertranslatable. For example, we have already seen that the theory of groups 1 and the theory of groups 2 are definitionally equivalent. Theorem 1 implies that they are intertranslatable too.

The converse of Theorem 1, however, does not hold. The following example provides a case of theories that are intertranslatable but not definitionally equivalent.

Example 6. Let $\Sigma=\{p\}$ be the signature containing a unary predicate symbol $p$. Consider the following two $\Sigma$-theories.

$$
\begin{aligned}
& T_{1}=\left\{\exists_{=1} x(x=x), \forall x p(x)\right\} \\
& T_{2}=\left\{\exists_{=1} x(x=x), \neg \forall x p(x)\right\}
\end{aligned}
$$

$T_{1}$ and $T_{2}$ are not definitionally equivalent since they do not have a common conservative extension. But $T_{1}$ and $T_{2}$ are intertranslatable. Consider the reconstrual $F: \Sigma \rightarrow \Sigma$ defined by letting $F p(x)$ be $\neg p(x)$. One can verify that $F: T_{1} \rightarrow T_{2}$ is a translation and that both

$$
\begin{aligned}
& T_{1} \vDash \forall x_{1} \ldots \forall x_{n}\left(\phi\left(x_{1}, \ldots, x_{n}\right) \leftrightarrow F F \phi\left(x_{1}, \ldots, x_{n}\right)\right) \\
& T_{2} \vDash \forall x_{1} \ldots \forall x_{n}\left(\phi\left(x_{1}, \ldots, x_{n}\right) \leftrightarrow F F \phi\left(x_{1}, \ldots, x_{n}\right)\right)
\end{aligned}
$$

hold for every $\Sigma$-formula $\phi\left(x_{1}, \ldots, x_{n}\right)$. This implies that $T_{1}$ and $T_{2}$ are intertranslatable.

Although the converse of Theorem 1 is not true in general, the following theorem establishes that it is true when one only considers theories in disjoint signatures.

Theorem 2. Let $\Sigma_{1}$ and $\Sigma_{2}$ be disjoint signatures with $T_{1}$ a $\Sigma_{1}$-theory and $T_{2}$ a $\Sigma_{2}$-theory. If $T_{1}$ and $T_{2}$ are intertranslatable, then they are definitionally equivalent.

Before proving Theorem 2 we need to do some work. Consider a translation $F: T_{1} \rightarrow T_{2}$ between a $\Sigma_{1}$-theory $T_{1}$ and a $\Sigma_{2}$-theory $T_{2}$. The translation $F$ gives rise to a map $F^{*}: \operatorname{Mod}\left(T_{2}\right) \rightarrow \operatorname{Mod}\left(T_{1}\right)$, which takes models of the theory $T_{2}$ to models of the theory $T_{1} \cdot{ }^{11}$ For every model $A$ of $T_{2}$ we first define a $\Sigma_{1}$-structure $F^{*}(A)$ as follows.

- $\operatorname{dom}\left(F^{*}(A)\right)=\operatorname{dom}(A)$.

- $\left(a_{1}, \ldots, a_{n}\right) \in p^{F^{*}(A)}$ if and only if $A \vDash F p\left[a_{1}, \ldots, a_{n}\right]$.

- $f^{F^{*}(A)}\left(a_{1}, \ldots, a_{n}\right)=b$ if and only if $A \vDash F f\left[a_{1}, \ldots, a_{n}, b\right]$.

\footnotetext{
${ }^{11}$ One can compare this with Gajda et al. (1987).
} 
- $c^{F^{*}(A)}=a$ if and only if $A \vDash F c[a]$.

One needs to verify that $f^{F^{*}(A)}$ and $c^{F^{*}(A)}$ are well-defined. For example, in the first case one needs to check that $f^{F^{*}(A)}$ is a function. We trivially know that $T_{1} \vDash \forall x_{1} \ldots \forall x_{n} \exists_{=1} y f\left(x_{1}, \ldots, x_{n}\right)=y$. Since $F$ is an interpretation, this implies that

$$
T_{2} \vDash F\left(\forall x_{1} \ldots \forall x_{n} \exists_{=1} y f\left(x_{1}, \ldots, x_{n}\right)=y\right)
$$

Unraveling the sentence on the right hand side, we see that this means that for all $a_{1}, \ldots, a_{n} \in F^{*}(A)$ there is a unique $b \in A$ such that $A \vDash F f\left[a_{1}, \ldots, a_{n}, b\right]$. So $f^{F^{*}(A)}$ is indeed a function. One goes through a similar argument to show that the element $c^{F^{*}(A)}$ is well-defined.

For every model $A$ of $T_{2}$ we have defined a $\Sigma_{1}$-structure $F^{*}(A)$. In order to show that $F^{*}(A)$ is actually a model of $T_{1}$ we need the following lemma.

Lemma. Let $A$ be a model of $T_{2}$ and $\phi\left(x_{1}, \ldots, x_{n}\right)$ a $\Sigma_{1}$-formula. Then $A \vDash$ $F \phi\left[a_{1}, \ldots, a_{n}\right]$ if and only if $F^{*}(A) \vDash \phi\left[a_{1}, \ldots, a_{n}\right]$.

Proof. By induction on the complexity of $\phi$.

Using the Lemma we can verify that for every model $A$ of $T_{2}, F^{*}(A)$ is a model of $T_{1}$. We let $\phi \in T_{1}$ be an axiom of $T_{1}$ and we show that $F^{*}(A) \vDash \phi$. Since $F$ is a translation, it must be that $T_{2} \vDash F \phi$, which means that $A \vDash F \phi$ since $A$ is a model of $T_{2}$. Then the Lemma implies that $F^{*}(A) \vDash \phi$. So indeed, $F^{*}(A)$ is a model of $T_{1}$ and we have successfully defined a $\operatorname{map} F^{*}: \operatorname{Mod}\left(T_{2}\right) \rightarrow \operatorname{Mod}\left(T_{1}\right)$ between models of $T_{2}$ and models of $T_{1}$.

We conclude our discussion of intertranslatability and definitional equivalence with a proof of Theorem 2 .

Proof of Theorem 2. Suppose that $T_{1}$ and $T_{2}$ are intertranslatable, with $F$ : $T_{1} \rightarrow T_{2}$ and $G: T_{2} \rightarrow T_{1}$ the relevant translations. We begin by defining definitional extensions $T_{1}^{+}$and $T_{2}^{+}$of $T_{1}$ and $T_{2}$ to the signature $\Sigma_{1} \cup \Sigma_{2}$.

We define $T_{1}^{+}=T_{1} \cup\left\{\delta_{s}: s \in \Sigma_{2}\right\}$, where for each symbol $s \in \Sigma_{2}$ the $\Sigma_{2^{-}}$ sentence $\delta_{s}$ is an explicit definition of $s$. If $q \in \Sigma_{2}$ is an $n$-ary predicate symbol then we let the definition $\delta_{q}$ be the sentence

$$
\forall x_{1} \ldots \forall x_{n}\left(q\left(x_{1}, \ldots, x_{n}\right) \leftrightarrow G q\left(x_{1}, \ldots, x_{n}\right)\right)
$$

If $g \in \Sigma_{2}$ is an $n$-ary function symbol then we let the definition $\delta_{g}$ be the sentence $\forall x_{1} \ldots \forall x_{n} \forall y\left(g\left(x_{1}, \ldots, x_{n}\right)=y \leftrightarrow G g\left(x_{1}, \ldots, x_{n}, y\right)\right)$. And if $d \in \Sigma_{2}$ is a constant symbol then we let $\delta_{d}$ be the sentence $\forall y(d=y \leftrightarrow G d(y))$. Using the Lemma one can verify that $T_{1}$ satisfies the admissibility conditions for $\delta_{g}$ and $\delta_{d}$.

We define $T_{2}^{+}=T_{2} \cup\left\{\delta_{t}: t \in \Sigma_{1}\right\}$ in the same manner. If $p \in \Sigma_{1}$ is an $n$-ary predicate symbol then we let the definition $\delta_{p}$ be the sentence

$$
\forall x_{1} \ldots \forall x_{n}\left(p\left(x_{1}, \ldots, x_{n}\right) \leftrightarrow F p\left(x_{1}, \ldots, x_{n}\right)\right)
$$

If $f \in \Sigma_{1}$ is an $n$-ary function symbol then we let the definition $\delta_{f}$ be the sentence $\forall x_{1} \ldots \forall x_{n} \forall y\left(f\left(x_{1}, \ldots, x_{n}\right)=y \leftrightarrow F f\left(x_{1}, \ldots, x_{n}, y\right)\right)$. If $c \in \Sigma_{1}$ is a 
constant symbol then we let $\delta_{c}$ be the sentence $\forall y(c=y \leftrightarrow F c(y))$. Using the above Lemma one can verify that $T_{2}$ satisfies the admissibility conditions for $\delta_{f}$ and $\delta_{c}$.

We show that $T_{1}^{+}$and $T_{2}^{+}$are logically equivalent. Without loss of generality, we show that every model of $T_{2}^{+}$is a model of $T_{1}^{+}$. The converse follows via an analogous argument. Let $A$ be a model of $T_{2}^{+}$. We show that $A$ is a model of $T_{1}^{+}$. There are two cases that need checking.

First, we show that $A \vDash \phi$ for every $\phi \in T_{1}$. We know that $F^{*}(A)$ is a model of $T_{1}$, so $F^{*}(A) \vDash \phi$. By the Lemma this means that $A \vDash F \phi$. One can verify by induction on the complexity of $\psi$ that for every model $A$ of $T_{2}^{+}$and every $\Sigma_{1}$-formula $\psi\left(x_{1}, \ldots, x_{n}\right)$,

$$
A \vDash \psi\left[a_{1}, \ldots, a_{n}\right] \text { iff } A \vDash F \psi\left[a_{1}, \ldots, a_{n}\right] .
$$

In particular, (7) implies that $A \vDash \phi$.

Second, we show that $A \vDash \delta_{s}$ for every $s \in \Sigma_{2}$. Let $q \in \Sigma_{2}$ be an $n$-ary predicate symbol. We show that $A \vDash \delta_{q}$. This follows since for all $a_{1}, \ldots, a_{n} \in A$

$$
A \vDash q\left[a_{1}, \ldots, a_{n}\right] \text { iff } A \vDash F G q\left[a_{1}, \ldots, a_{n}\right] \text { iff } A \vDash G q\left[a_{1}, \ldots, a_{n}\right]
$$

The first equivalence follows from condition (4) in the definition of intertranslatability and the fact that $A$ is a model of $T_{2}^{+}$. The second equivalence follows from (7). This string of equivalences implies that $A \vDash \delta_{q}$. In a similar manner one can verify that $A \vDash \delta_{g}$ for every function symbol $g \in \Sigma_{2}$ and that $A \vDash \delta_{d}$ for every constant symbol $d \in \Sigma_{2}$.

We have therefore shown that $A$ is a model of $T_{2}^{+}$. We conclude that $T_{1}^{+}$ and $T_{2}^{+}$are logically equivalent, so $T_{1}$ and $T_{2}$ are definitionally equivalent.

\section{Conclusion}

It is important to note that intertranslatability does not suffer from the same shortcomings as Quine's original criterion. Theorem 2 implies that the two theories from Example 5 are not intertranslatable. Example 4 does not pose a problem for intertranslatability either. One can easily verify that if $T_{1}$ and $T_{2}$ are intertranslatable, then $T_{1}$ is complete if and only if $T_{2}$ is complete. So the two theories in Example 4 are not intertranslatable.

Quine originally considered theoretical equivalence because of its relationship to underdetermination. ${ }^{12}$ Our discussion of Quine equivalence yields a small remark concerning this relationship. A theory $T$ is underdetermined if there is another theory $T^{\prime}$ such that (i) $T$ and $T^{\prime}$ are empirically equivalent but (ii) $T$ and $T^{\prime}$ are theoretically inequivalent. Quine proposed Quine equivalence as a way to make condition (ii) precise. We have shown here, however, that it is too liberal a criterion for theoretical equivalence. According to Quine equivalence, there are few pairs of theories that satisfy (ii), and so there are few

\footnotetext{
${ }^{12}$ See (Quine, 1975, 318-322) and Coffey (2014).
} 
cases of underdetermination. If one takes Quine equivalence as the standard for theoretical equivalence, one underestimates the threat of underdetermination.

Our discussion also allows one to evaluate Quine's criterion against Glymour's. Theorems 1 and 2 demonstrate a natural and precise sense in which definitional equivalence is an amendment to Quine equivalence. Quine's original criterion for theoretical equivalence was unsatisfactory, and when it is fixed it becomes essentially the same as Glymour's criterion.*

\section{References}

Andréka, H., Madarász, J. X., and Németi, I. (2005). Mutual definability does not imply definitional equivalence, a simple example. Mathematical Logic Quarterly.

Barrett, T. W. (2014). On the structure of classical mechanics. The British Journal for the Philosophy of Science (forthcoming).

Coffey, K. (2014). Theoretical equivalence as interpretive equivalence. The British Journal for the Philosophy of Science.

Curiel, E. (2014). Classical mechanics is Lagrangian; it is not Hamiltonian. The British Journal for the Philosophy of Science.

de Bouvére, K. L. (1965). Synonymous theories. In Symposium on the Theory of Models. North-Holland Publishing Company.

Friedman, H. and Visser, A. (2014). When bi-interpretability implies synonymy. Manuscript.

Gajda, A., Krynicki, M., and Szczerba, L. (1987). A note on syntactical and semantical functions. Studia Logica.

Glymour, C. (1970). Theoretical realism and theoretical equivalence. In PSA: Proceedings of the Biennial Meeting of the Philosophy of Science Association.

Glymour, C. (1977). The epistemology of geometry. Nous.

Glymour, C. (1980). Theory and Evidence. Princeton.

Glymour, C. (2013). Theoretical equivalence and the semantic view of theories. Philosophy of Science.

Halvorson, H. (2011). Natural structures on state space. Manuscript.

Halvorson, H. (2012). What scientific theories could not be. Philosophy of Science.

* This material is based upon work supported by the National Science Foundation under Grant No. DGE 1148900. 
Halvorson, H. (2013). The semantic view, if plausible, is syntactic. Philosophy of Science.

Hodges, W. (2008). Model Theory. Cambridge University Press.

Kanger, S. (1968). Equivalent theories. Theoria.

Knox, E. (2013). Newtonian spacetime structure in light of the equivalence principle. The British Journal for the Philosophy of Science.

North, J. (2009). The 'structure' of physics: A case study. Journal of Philosophy.

Pelletier, F. J. and Urquhart, A. (2003). Synonymous logics. Journal of Philosophical Logic.

Quine, W. V. O. (1975). On empirically equivalent systems of the world. Erkenntnis.

Sklar, L. (1982). Saving the noumena. Philosophical Topics.

Swanson, N. and Halvorson, H. (2012). On North's 'The structure of physics'. Manuscript.

van Fraassen, B. C. (2014). One or two gentle remarks about Hans Halvorson's critique of the semantic view. Manuscript.

Visser, A. (2006). Categories of theories and interpretations. In Logic in Tehran. Proceedings of the workshop and conference on Logic, Algebra and Arithmetic, held October 18-22, 2003. ASL.

Weatherall, J. O. (2014). Are Newtonian gravitation and geometrized Newtonian gravitation theoretically equivalent? Manuscript. 